\title{
The effect of exercise on maternal complications and birth outcomes in overweight or obese pregnant women: a meta-analysis
}

\author{
Yu Xing, Xin Wang, Weiyuan Zhang, Haili Jiang \\ Obstetrics Department, Beijing Obstetrics and Gynecology Hospital, Capital Medical University, Beijing, China \\ Contributions: (I) Conception and design: Y Xing, H Jiang; (II) Administrative support: H Jiang; (III) Provision of study materials or patients: Y Xing, \\ X Wang, W Zhang; (IV) Collection and assembly of data: Y Xing, X Wang, W Zhang; (V) Data analysis and interpretation: Y Xing; (VI) Manuscript \\ writing: All authors; (VII) Final approval of manuscript: All authors. \\ Correspondence to: Haili Jiang, MM. Obstetrics Department, Beijing Obstetrics and Gynecology Hospital, Capital Medical University, No. 251 \\ Yaojiayuan Road, Chaoyang District, Beijing 100026, China. Email: jianghaili2020@ccmu.edu.cn
}

\begin{abstract}
Background: Overweight and obesity can increase the risk for certain adverse obstetric outcomes. Exercise may reduce these risks by promoting weight loss or preventing excessive weight gain. Therefore, this study aims to evaluate the effect of exercise therapy on pregnancy complications and birth outcomes in overweight or obese pregnant women through a meta-analysis.
\end{abstract}

Methods: We searched the electronic databases PubMed, Cochrane Library, Embase, and Web of Science for studies on the effects of exercise therapy on pregnancy complications and birth outcomes in overweight or obese pregnant women. The data were analyzed using Stata software for meta-analysis.

Results: A total of 1,709 pregnant women who met the eligibility criteria were included across 13 studies. The meta-analysis demonstrated a significant difference between the exercise group and the control group in regards to gestational weight gain [GWG; standard mean difference (SMD): $-0.21,95 \%$ confidence interval (CI): -0.32 to -0.10$)$ ] and gestational hypertension [GH; risk ratio (RR) and 95\% CI were $0.53(0.32-0.88)]$. Contrastingly, no significant differences were found between the 2 groups in terms of the other evaluation indicators, including gestational length at delivery, Apgar score at 1 minute and 5 minutes, gestational diabetes, cesarean delivery, preterm delivery, preeclampsia, macrosomia, and neonatal asphyxia.

Conclusions: The results of this study suggest that exercise can reduce the risk of GWG and the occurrence rate of GH in overweight or obese pregnant women, however, exercise had no effect on birth outcomes.

Keywords: Exercise; obese; overweight; pregnancy

Submitted Sep 20, 2020. Accepted for publication Nov 12, 2020.

doi: 10.21037/apm-20-2097

View this article at: http://dx.doi.org/10.21037/apm-20-2097

\section{Introduction}

The incidence of overweight and obesity has risen significantly in the last several decades. From 1980 to 2013, overweight and obesity has increased about $10 \%$ in women globally (1). Approximately 1 in 4 women are overweight after childbirth and 1 in 5 women are obese before pregnancy (2). Women of childbearing age should therefore strengthen their nutrition during this special physiological process. The lack of nutritional knowledge and blind supplementation can not only result in unnecessary obesity, but can also cause nutritional imbalances, and result in unnecessary risks to pregnancy and delivery (3-5).

Overweight or obesity can increase the risk for adverse obstetric outcomes, which may increase the risk of neonatal mortality $(6,7)$ as well as gestational hypertension (GH), preeclampsia, gestational diabetes, cesarean delivery, 
neonatal intensive care unit admission, extended length of hospital stays, large for gestational age infants, and neonatal asphyxia (8-11). In addition, children born to obese pregnant women have a relatively higher risk of cardiovascular disease in later life compared to those born to normal weight women $(12,13)$.

Pregnancy-induced hypertension is one of the most common pregnancy complications, which causes serious damage to the heart, brain, kidney, liver, and other important organs in pregnant women, and poses a significant threat to mothers and infants (14-16). The increased blood glucose of overweight or obese pregnant women leads to an increase in fetal blood glucose and an increase in insulin secretion by fetal islet $\beta$ cells stimulated by fetal hyperglycemia (17-20). Fetal insulin mainly promotes fetal growth and promotes weight gain, which may lead to macrosomia.

Exercise is universally acknowledged as a way to stay healthy and to reduce pregnancy complications and adverse birth outcomes by promoting weight loss or preventing weight gain (21). However, the impact of exercise on overweight and obese pregnant women is controversial.

There are several articles analyzing the association between overweight and obesity in pregnancy with pregnancy complications and birth outcomes, in which there exist various research designs, recruitment and exclusion criteria, methods, and results. Davenport et al. (22) performed a meta-analysis in 2018 which contained all study design with considerable heterogeneity. For the reason, a meta-analysis was performed in this study only included randomized controlled trials to evaluate the effect of exercise on pregnancy complications and birth outcomes in overweight or obese pregnant women. We present the following article in accordance with the PRISMA reporting checklist (available at http://dx.doi.org/10.21037/apm-20-2097).

\section{Methods}

\section{Searched databases and strategies}

We searched PubMed, Cochrane Library, Embase, and Web of Science using the terms "pregnancy" combined with "obese" or "overweight" and "complications" or "outcome" and "exercise" between January 1974 and January 2020. Two independent investigators conducted a preliminary survey, deleted duplicate records, screened the relevance of titles and summaries, and determined which publications were excluded or required further assessment. We then reviewed the full text for inclusion. We also manually checked the references of the retrieved articles and previous reviews to identify additional eligible studies. In order to obtain more relevant research and higher accuracy, the reference list of each article retrieved was also reviewed.

\section{Inclusion and exclusion criteria}

Studies were included if:

(I) They were randomized controlled trials (RCTs);

(II) Studied the effect of exercise for overweight or obese pregnant women;

(III) Involved no less than one evaluation indicator;

(IV) English language only.

Studies were excluded if:

(I) They were case studies/meta-analyses/letter to editors;

(II) Included patients with other diseases;

(III) Duplicate publications

The Grading of Recommendations Assessment, Development and Evaluation (GRADE) framework was used to assess the quality of included studies. Accordingly, evidence from RCTs was considered high quality.

\section{Data extraction and review}

Two reviewers independently extracted the data and full text. For each study, the data collected included date of publication, first author, nation, number of patients enrolled and randomized in each study, age (years), recruitment gestational week and BMI, and intervention measures and outcomes. Literature quality of RCTs was assessed using the Jadad score, where a score of 1-2 was low quality, and a score of $3-5$ was high quality.

\section{Evaluation indicator}

Gestational diabetes, GH, preeclampsia, cesarean delivery, gestational weight gain (GWG), gestational length at delivery, preterm delivery, Apgar score at 1 and 5 minutes, macrosomia, and neonatal asphyxia were extracted as the evaluation indicators in this study.

\section{Statistical analysis}

Statistical software Stata (SE) 11.2 was used to analyze the selected literature. A heterogeneity test was conducted before data consolidation. If $\mathrm{P}>0.05$ or $\mathrm{I}^{2}<50 \%$, the data 


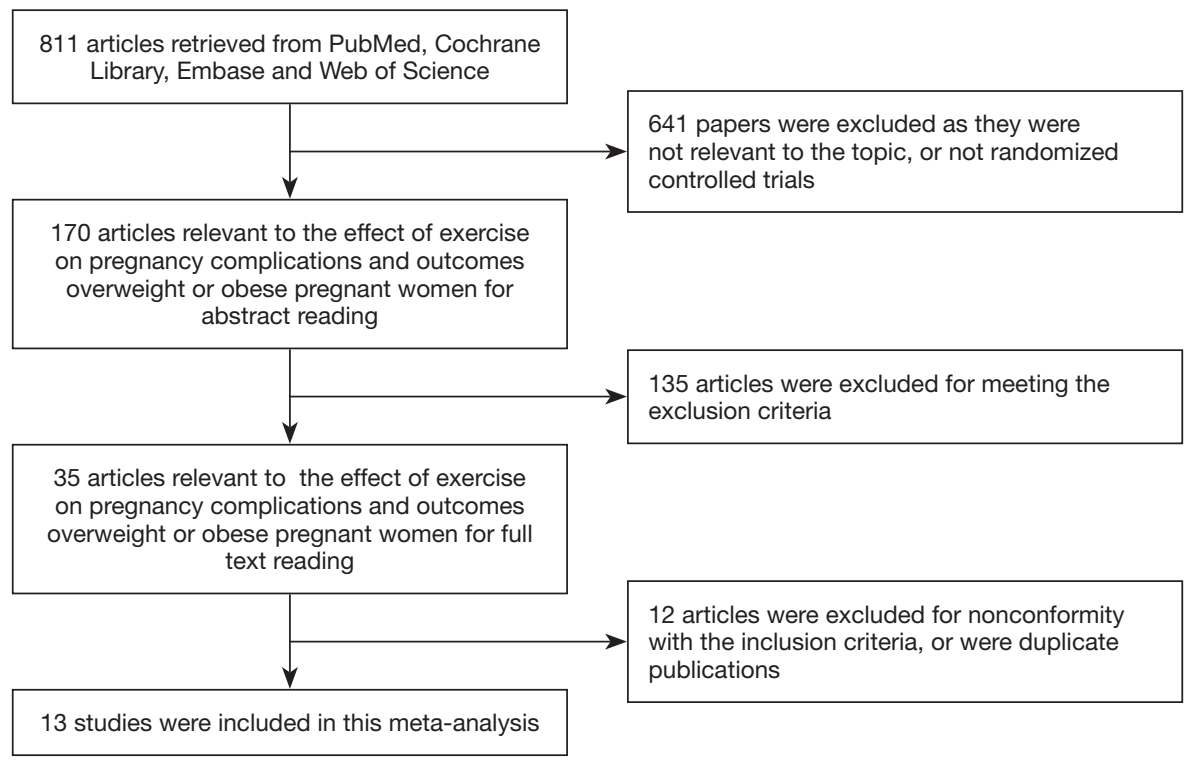

Figure 1 Flow diagram of the search process, study identification, and study inclusion and exclusion.

was considered to be homogeneous, and the fixed effect model was adopted; otherwise, the random effect model was adopted. The count data were represented by relative risk (RR) as the effect quantity, and the measurement data were represented by standard mean difference (SMD), both of which gave $95 \%$ confidence intervals (CI). Publication bias was concomitantly examined by Begg's test.

\section{Results}

\section{Search results}

The electronic database search resulted in a total number of 811 articles. After thorough review, 13 papers (23-35) eventually met all inclusion criteria. The other 798 articles were excluded due to repetition, irrelevant studies, no control groups, incomplete data or comparisons, other operations, reviews, and incomplete articles. Figure 1 is a flowchart of identification, inclusion, and exclusion, reflecting the search process and the reasons for exclusion. Of all the included studies, the Jadad scores of 11 studies were equal to or greater than 3 , and the other 2 papers were of low quality.

\section{Main features of the studies}

Table 1 summarizes the characteristics of the included studies. This analysis consisted of a total number of 1,709 patients. All articles were published from 2009 to 2017. This study included 859 patients in the exercise group and 850 patients in the control group.

\section{Results of the meta-analysis}

\section{Meta-analysis on GWG}

A total of 12 studies with 709 patients in the exercise group and 696 patients in the control group involved GWG. Figure 2 shows the forest plot of the summarized results. All 12 studies showed significant differences in GWG between the 2 groups (SMD $=-0.21,95 \%$ CI: $-0.32--0.10$, $\mathrm{P}<0.001$; $\mathrm{P}$ for heterogeneity $\left.=0.075, \mathrm{I}^{2}=39.9 \%\right)$. GWG in the exercise group was significantly lower compared to the control group.

\section{Meta-analysis on gestational diabetes}

The forest plot for the meta-analysis on gestational diabetes is presented in Figure 3. The results demonstrated no significant differences between the exercise group with 794 patients and the control group with 786 patients $(\mathrm{RR}=0.71$, 95\% CI: $0.48-1.04, \mathrm{P}=0.081$; $\mathrm{P}$ for heterogeneity $=0.004$, $\mathrm{I}^{2}=63.1 \%$, the random effect model was adopted).

\section{Meta-analysis on GH}

A total of 6 studies involved data on GH. The forest plot for the meta-analysis on GH is shown in Figure 4. The occurrence of $\mathrm{GH}$ in the exercise group (428 patients) 


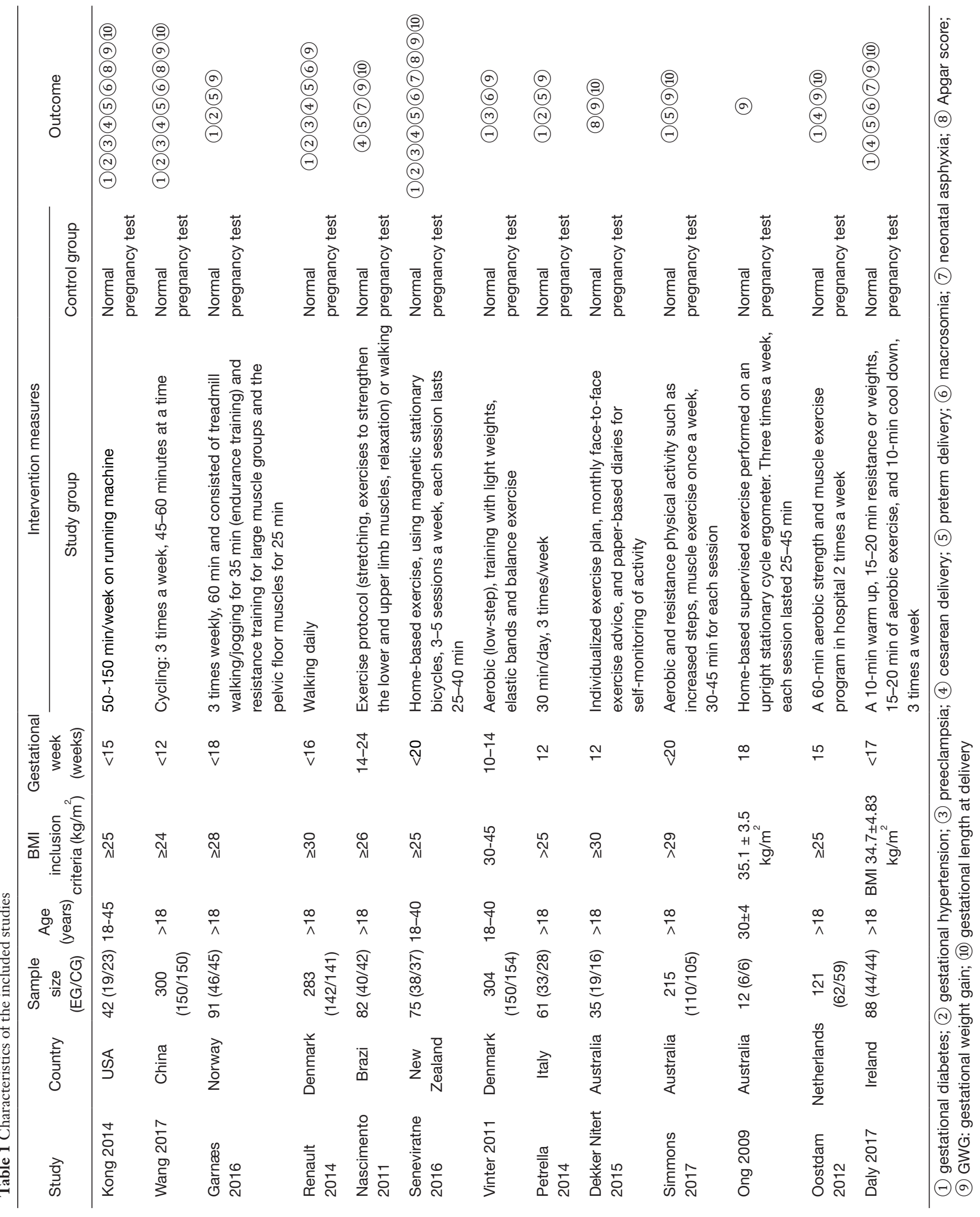




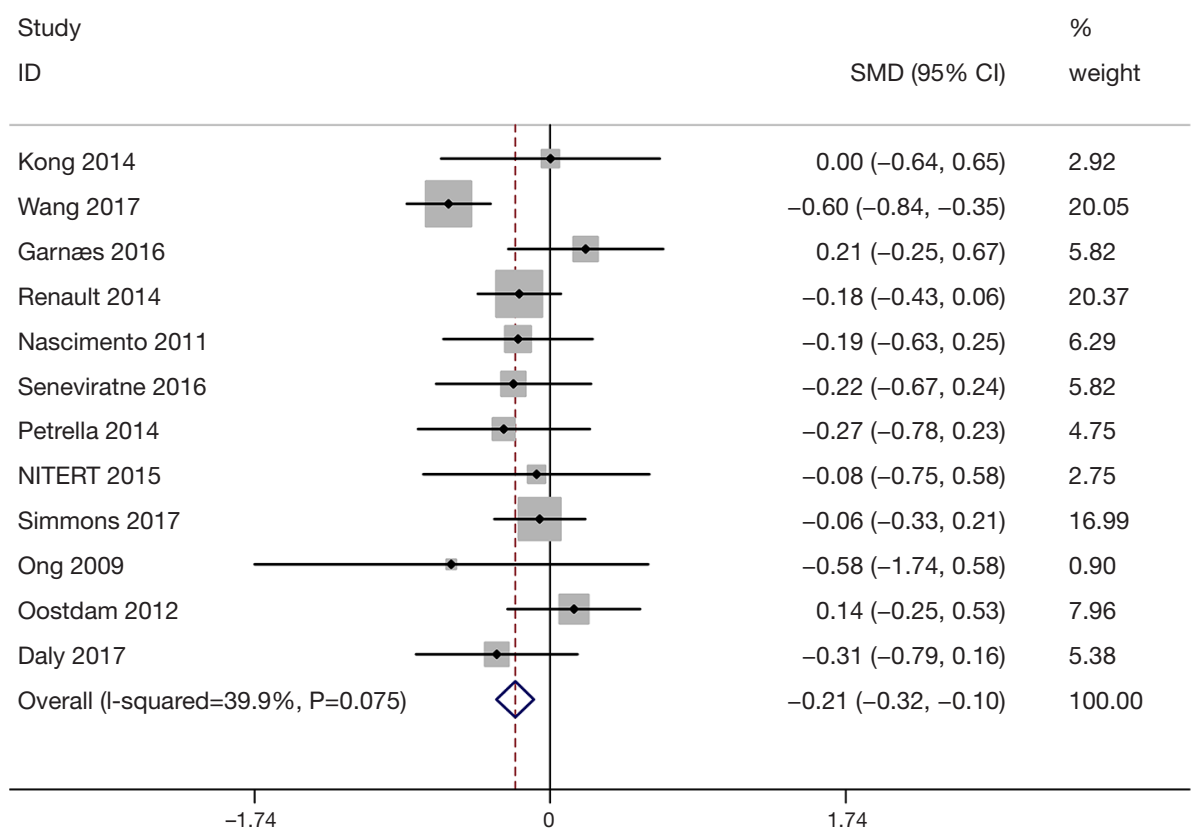

Figure 2 Forest plot for gestational weight gain (GWG) in the exercise group and the control group. SMD, standard mean difference.

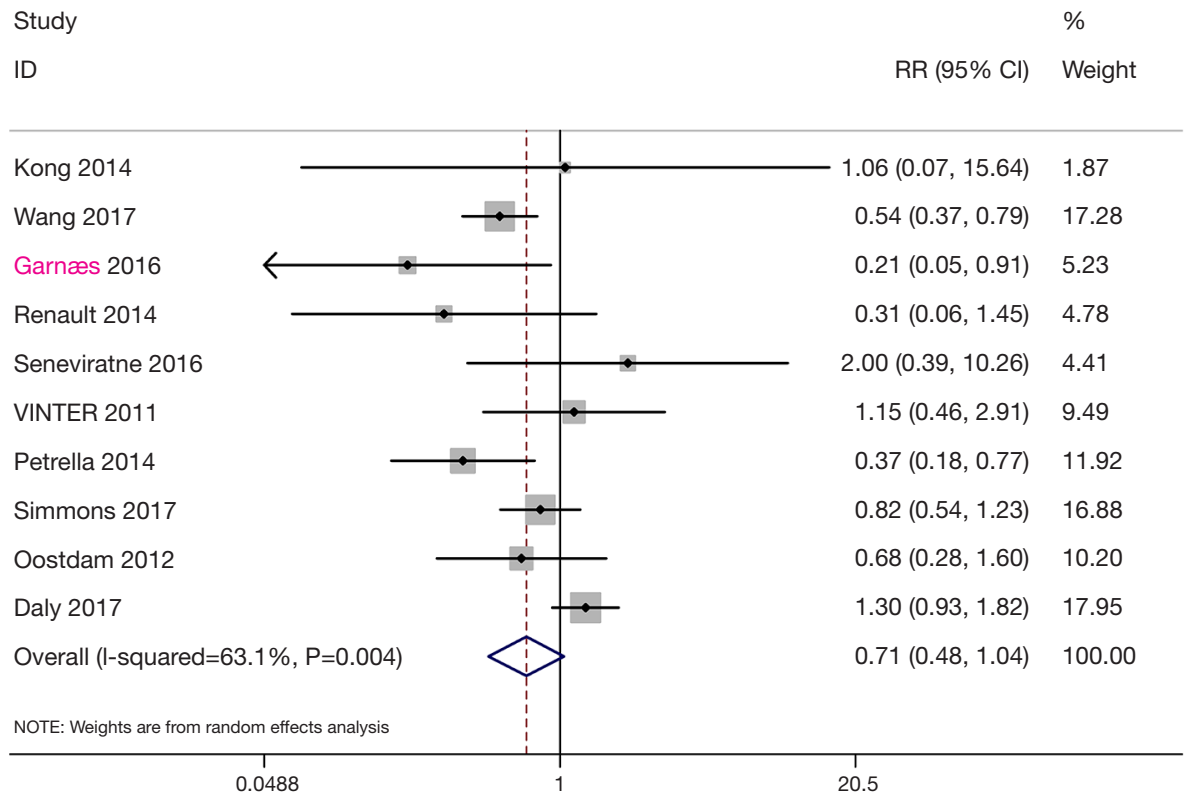

Figure 3 Forest plot for gestational diabetes in the exercise group and the control group. RR, relative risk. 


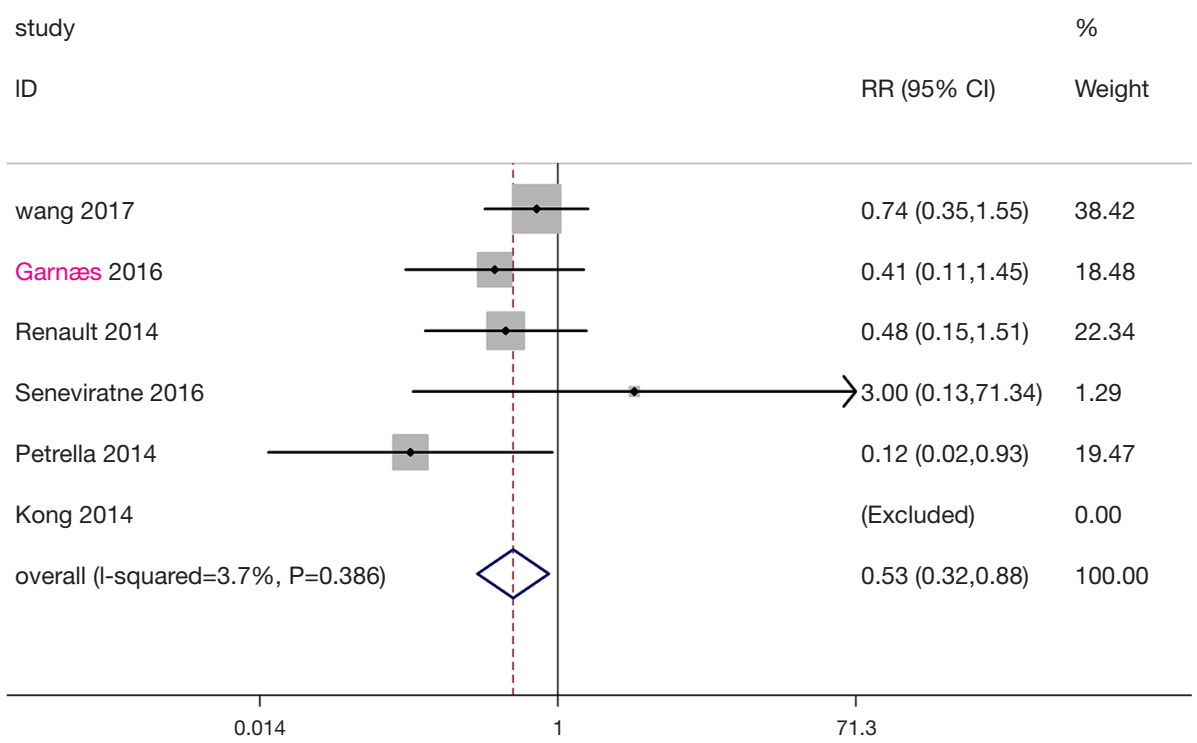

Figure 4 Forest plot for gestational hypertension $(\mathrm{GH})$ in the exercise group and the control group. RR, relative risk.

Table 2 Meta-analysis results for the other evaluation indicators

\begin{tabular}{lcccc}
\hline Evaluation indicator & Samples (exercise group/control group) & RR/SMD & $95 \% \mathrm{Cl}$ & Heterogeneity \\
\hline Gestational length at delivery (week) & $482 / 476$, study $(\mathrm{n})=8$ & -0.05 & $-0.19-0.08$ & $\mathrm{I}^{2}=0.0 \%, \mathrm{P}=0.565$ \\
Apgar score 1 & $207 / 210$, study $(\mathrm{n})=3$ & 0.18 & $-0.02-0.38$ & $\mathrm{I}^{2}=0.0 \%, \mathrm{P}=0.972$ \\
Apgar score 5 & $226 / 226$, study $(\mathrm{n})=4$ & 0.12 & $-0.20-0.45$ & $\mathrm{I}^{2}=0.0 \%, \mathrm{P}=0.860$ \\
Cesarean delivery & $495 / 496$, study $(\mathrm{n})=7$ & 0.99 & $0.84-1.17$ & $\mathrm{I}^{2}=0.0 \%, \mathrm{P}=0.708$ \\
Preterm delivery & $622 / 615$, study $(\mathrm{n})=9$ & 0.74 & $0.45-1.24$ & $\mathrm{I}^{2}=5.1 \%, \mathrm{P}=0.392$ \\
Preeclampsia & $499 / 505$, study $(\mathrm{n})=5$ & 1.00 & $0.66-1.52$ & $\mathrm{I}^{2}=0.0 \%, \mathrm{P}=0.793$ \\
Macrosomia & $543 / 549$, study $(\mathrm{n})=6$ & 1.13 & $0.88-1.45$ & $\mathrm{I}^{2}=0.0 \%, \mathrm{P}=0.883$ \\
Neonatal asphyxia & $122 / 123$, study $(\mathrm{n})=3$ & 0.86 & $0.26-2.90$ & $\mathrm{I}^{2}=0.0 \%, \mathrm{P}=0.643$ \\
\hline
\end{tabular}

$\mathrm{RR}$, relative risk; SMD, standard mean difference.

was lower than that in the control group (424 patients) $(\mathrm{RR}=0.53,95 \% \mathrm{CI}: 0.32-0.88, \mathrm{P}=0.014$; P for heterogeneity $\left.=0.386, \mathrm{I}^{2}=3.7 \%\right)$.

\section{Other results}

Meta-analysis results for other evaluation indicators are shown in Table 2. The meta-analysis results of other indicators including preeclampsia, cesarean delivery, gestational length at delivery, preterm delivery, Apgar score at 1 and 5 minutes, macrosomia, and neonatal asphyxia in the exercise group were similar to those of the control group.

\section{Sensitivity analysis}

We analyzed the data after excluding studies one by one, and there was no significant difference between the statistical results after exclusion and those before exclusion, indicating that the research results were relatively stable and reliable (Figure 5).

\section{Bias analysis}

All studies on GWG were included in the funnel plot. The results showed that the funnel plot had good symmetry 


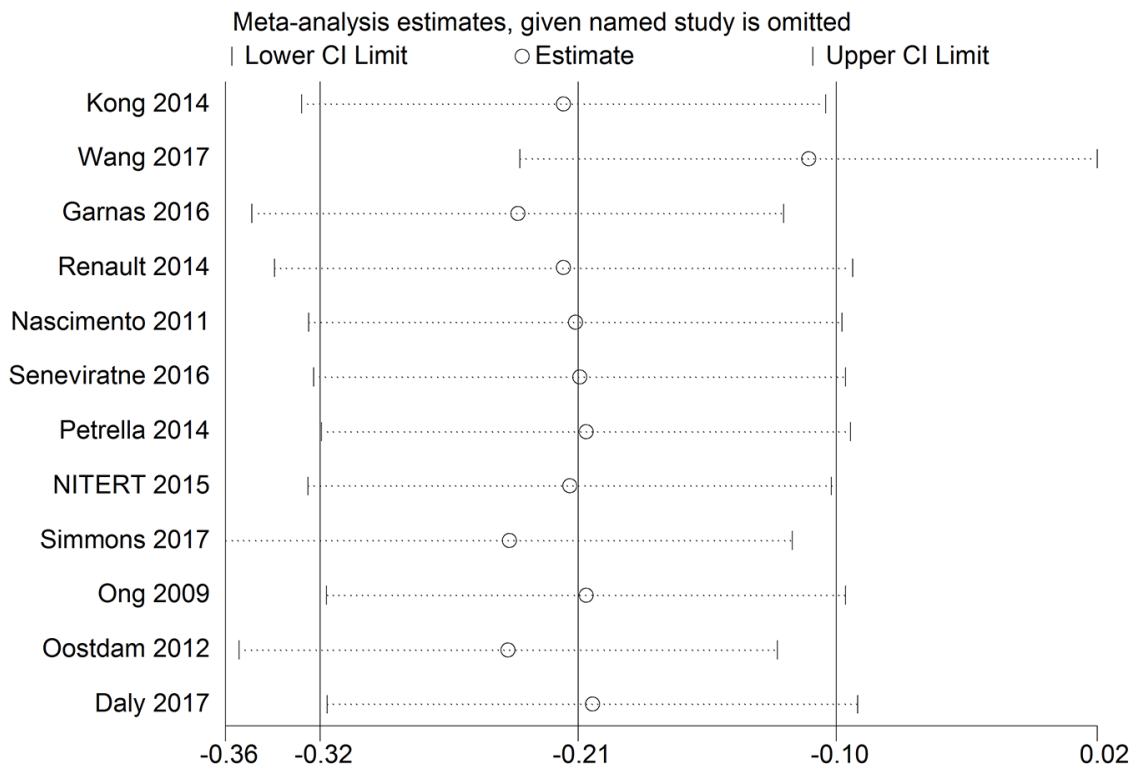

Figure 5 Sensitivity analysis of gestational weight gain (GWG) in the exercise group and the control group. CI, confidence interval.

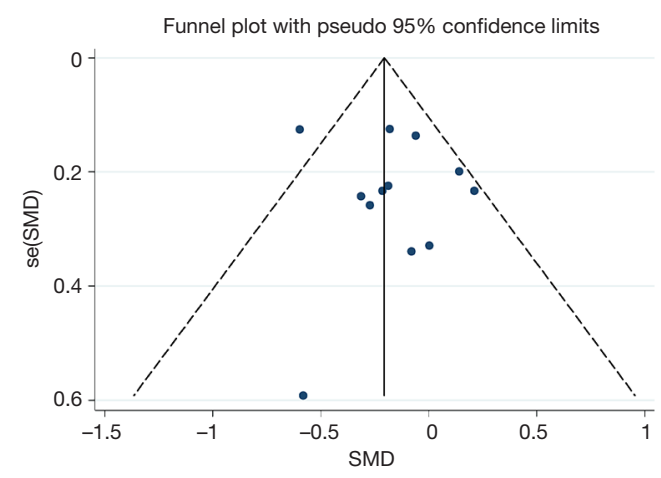

Figure 6 Funnel plot of publication bias in studies on gestational weight gain (GWG). SMD, standard mean difference.

and little publication bias (Figure 6). The result of Begg's test suggested that no significant evidence of potential publication bias existed $(\mathrm{z}=0.48, \mathrm{P}=0.631)$.

\section{Discussion}

This meta-analysis of 13 RCTs including 1,709 women showed that exercise in overweight or obese pregnancies could reduce GWG by $0.21 \mathrm{~kg}$, and lower the risk of GH by $47 \%$. However, there was no significant effect of exercise on other important clinical maternal and infant outcomes including gestational diabetes, preeclampsia, cesarean delivery, gestational length at delivery, preterm delivery, Apgar score at 1 and 5 minutes, macrosomia, and neonatal asphyxia.

Excessive GWG has been reported to increase the risk of poor prognosis for pregnancy outcomes, which may result in detrimental consequences for both maternal and infant health $(4,36)$. Previous studies report a generally positive effect of exercise on GWG in pregnant women, although results differ between studies. A meta-analysis conducted by Streuling et al. concluded that exercise reduced GWG by $0.61 \mathrm{~kg}$ in pregnant women (37), while Du et al. demonstrated that physical exercise reduced GWG by $1.14 \mathrm{~kg}$ (38). This is consistent with our results, demonstrating a $0.21 \mathrm{~kg}$ reduction through exercise during pregnancy.

Several previous studies or systematic reviews and metaanalyses have reported the effect of exercise on gestational diabetes, however, the results are inconclusive. A metaanalysis found that physical exercise significantly reduced the risk of gestational diabetes (39) $(\mathrm{RR}=0.69,95 \% \mathrm{CI}$ : 0.52-0.91), and other studies found that exercise could lower the risk of gestational diabetes by $39 \%$ (40) and by $29 \%$ (38) in overweight and obese pregnant women. Nevertheless, some previous studies have demonstrated contrasting results $(41,42)$, consistent with the results in our study, no significant differences between the exercise group and the control group on gestational diabetes incidence. Due to the heterogeneity in diagnostic criteria for 
gestational diabetes among studies, this discordance could influence the pooled effect.

Contrary to the negative results of several studies focused on exercise for overweight or obese pregnant women (41-43), the present study found a significant reduction in the risk of $\mathrm{GH}$ in the exercise group compared to the control group ( $\mathrm{RR}=0.53,95 \% \mathrm{CI}: 0.32-0.88)$. This way of movement is simple, economical, and most pregnant women can perform, hence, exercise should be actively promoted during pregnancy.

With respect to results regarding other complications and infant outcomes, we did not observe any differences between the exercise group and the control group. A metaanalysis showed that supervised prenatal exercise reduced the risks of large for gestational age and macrosomia without an increased risk of small for gestational age (21), and another recent meta-analysis found that exercise reduced the risk of preterm birth by $38 \%$ ( $R R=0.62,95 \%$ CI: 0.41-0.95) (40). Our current study found no significant effect of exercise during pregnancy in overweight or obese pregnant women on preeclampsia, cesarean delivery, gestational length at delivery, preterm delivery, Apgar score at 1 minute and 5 minutes, macrosomia, and neonatal asphyxia, which is consistent with other studies $(13,44,45)$.

This study has some limitations. Firstly, it is difficult to maintain consistency across exercise patterns, duration, and intensity across the original included studies. Secondly, the baseline BMI was inconsistent across all studies. In addition, selection bias might have been introduced due to the inclusion of articles only published in English.

In conclusion, current evidence shows that exercise therapy has a beneficial effect on pregnancy complications and outcomes in overweight or obese pregnant women, and can effectively reduce the occurrence of GH and GWG. We may suggest that aerobic exercise for $30 \mathrm{~min}$ to 1 hour, three times a week may be beneficial to pregnant women. However, there is insufficient evidence to support superior birth outcomes, requiring further well-designed clinical studies to explore the relationship between exercise and pregnancy complications and birth outcomes in the future.

\section{Acknowledgments}

Funding: None.

\section{Footnote}

Reporting Checklist: The authors have completed the
PRISMA reporting checklist. Available at http://dx.doi. org/10.21037/apm-20-2097

Conflicts of Interest: All authors have completed the ICMJE uniform disclosure form (available at http://dx.doi. org/10.21037/apm-20-2097). The authors have no conflicts of interest to declare.

Etbical Statement: The authors are accountable for all aspects of the work in ensuring that questions related to the accuracy or integrity of any part of the work are appropriately investigated and resolved.

Open Access Statement: This is an Open Access article distributed in accordance with the Creative Commons Attribution-NonCommercial-NoDerivs 4.0 International License (CC BY-NC-ND 4.0), which permits the noncommercial replication and distribution of the article with the strict proviso that no changes or edits are made and the original work is properly cited (including links to both the formal publication through the relevant DOI and the license). See: https://creativecommons.org/licenses/by-nc-nd/4.0/.

\section{References}

1. Ng M, Fleming T, Robinson M, et al. Global, regional, and national prevalence of overweight and obesity in children and adults during 1980-2013: a systematic analysis for the Global Burden of Disease Study 2013. Lancet 2014;384:766-81.

2. Robbins CL, Zapata LB, Farr SL, et al. Core state preconception health indicators - pregnancy risk assessment monitoring system and behavioral risk factor surveillance system, 2009. MMWR Surveill Summ 2014;63:1-62.

3. Kriebs JM. Obesity as a complication of pregnancy and labor. J Perinat Neonatal Nurs 2009;23:15-22.

4. Baeten JM, Bukusi EA, Lambe M. Pregnancy complications and outcomes among overweight and obese nulliparous women. Am J Public Health 2001;91:436-40.

5. Guelinckx I, Devlieger R, Beckers K, et al. Maternal obesity: pregnancy complications, gestational weight gain and nutrition. Obes Rev 2008;9:140-50.

6. Nohr EA. Maternal obesity and neonatal mortality in an African setting. Lancet 2012;380:1292-3.

7. Johansson S, Villamor E, Altman M, et al. Maternal overweight and obesity in early pregnancy and risk of infant mortality: a population based cohort study in 
Sweden. BMJ 2014;349:g6572.

8. Gaillard R, Durmuş B, Hofman A, Mackenbach JP, Steegers EA, Jaddoe VW: Risk factors and outcomes of maternal obesity and excessive weight gain during pregnancy. Obesity (Silver Spring) 2013;21:1046-55.

9. Crane JM, Murphy P, Burrage L, et al. Maternal and perinatal outcomes of extreme obesity in pregnancy. $\mathrm{J}$ Obstet Gynaecol Can 2013;35:606-11.

10. Pantham P, Aye IL, Powell TL. Inflammation in maternal obesity and gestational diabetes mellitus. Placenta 2015;36:709-15.

11. Foo XY, Greer RM, Kumar S. Impact of Maternal Body Mass Index on Intrapartum and Neonatal Outcomes in Brisbane, Australia, 2007 to 2013. Birth 2016;43:358-65.

12. Lee KK, Raja EA, Lee AJ, et al. Maternal Obesity During Pregnancy Associates With Premature Mortality and Major Cardiovascular Events in Later Life. Hypertension 2015;66:938-44.

13. Pugh SJ, Hutcheon JA, Richardson GA, et al. Child academic achievement in association with pre-pregnancy obesity and gestational weight gain. J Epidemiol Community Health 2016;70:534-40.

14. Edwards LE, Hellerstedt WL, Alton IR, et al. Pregnancy complications and birth outcomes in obese and normalweight women: effects of gestational weight change. Obstet Gynecol 1996;87:389-94.

15. Ramachenderan J, Bradford J, McLean M. Maternal obesity and pregnancy complications: a review. Aust N Z J Obstet Gynaecol 2008;48:228-35.

16. Abrams B, Parker J. Overweight and pregnancy complications. Int J Obes 1988;12:293-303.

17. Marshall NE, Spong CY. Obesity, pregnancy complications, and birth outcomes. Semin Reprod Med 2012;30:465-71.

18. Santangelo C, Varì R, Scazzocchio B, et al. Management of reproduction and pregnancy complications in maternal obesity: which role for dietary polyphenols? Biofactors 2014;40:79-102.

19. Mutsaerts MA, Groen H, Buiter-Van der Meer A, et al. Effects of paternal and maternal lifestyle factors on pregnancy complications and perinatal outcome. A population-based birth-cohort study: the GECKO Drenthe cohort. Hum Reprod 2014;29:824-34.

20. Mahizir D, Briffa JF, Hryciw DH, et al. Maternal obesity in females born small: Pregnancy complications and offspring disease risk. Mol Nutr Food Res 2016;60:8-17.

21. Artal R. Exercise in Pregnancy: Guidelines. Clin Obstet Gynecol 2016;59:639-44.
22. Davenport MH, Meah VL, Ruchat SM, et al. Impact of prenatal exercise on neonatal and childhood outcomes: a systematic review and meta-analysis. Br J Sports Med 2018;52:1386-96.

23 Wang C, Wei Y, Zhang X, et al. A randomized clinical trial of exercise during pregnancy to prevent gestational diabetes mellitus and improve pregnancy outcome in overweight and obese pregnant women. Am J Obstet Gynecol 2017;216:340-51.

24. Kong KL, Campbell CG, Foster RC, et al. A pilot walking program promotes moderate-intensity physical activity during pregnancy. Med Sci Sports Exerc 2014;46:462-71.

25. Garnæs KK, Mørkved S, Salvesen Ø, et al. Exercise Training and Weight Gain in Obese Pregnant Women: A Randomized Controlled Trial (ETIP Trial). PLoS Med 2016;13:e1002079.

26. Renault KM, Nørgaard K, Nilas L, et al. The Treatment of Obese Pregnant Women (TOP) study: a randomized controlled trial of the effect of physical activity intervention assessed by pedometer with or without dietary intervention in obese pregnant women. Am J Obstet Gynecol 2014;210:134.e1-9.

27. Nascimento SL, Surita FG, Parpinelli M, et al. The effect of an antenatal physical exercise programme on maternal/ perinatal outcomes and quality of life in overweight and obese pregnant women: a randomised clinical trial. BJOG 2011;118:1455-63.

28. Seneviratne SN, Jiang Y, Derraik J, et al. Effects of antenatal exercise in overweight and obese pregnant women on maternal and perinatal outcomes: a randomised controlled trial. BJOG 2016;123:588-97.

29. Vinter CA, Jensen DM, Ovesen P, et al. The LiP (Lifestyle in Pregnancy) study: a randomized controlled trial of lifestyle intervention in 360 obese pregnant women. Diabetes Care 2011;34:2502-7.

30. Petrella E, Malavolti M, Bertarini V, et al. Gestational weight gain in overweight and obese women enrolled in a healthy lifestyle and eating habits program. J Matern Fetal Neonatal Med 2014;27:1348-52.

31. Dekker Nitert M, Barrett HL, Denny KJ, et al. Exercise in pregnancy does not alter gestational weight gain, MCP1 or leptin in obese women. Aust N Z J Obstet Gynaecol 2015;55:27-33.

32. Simmons D, Devlieger R, van Assche A, et al. Effect of Physical Activity and/or Healthy Eating on GDM Risk: The DALI Lifestyle Study. J Clin Endocrinol Metab 2017;102:903-13.

33. Ong MJ, Guelfi KJ, Hunter T, et al. Supervised home- 
based exercise may attenuate the decline of glucose tolerance in obese pregnant women. Diabetes Metab 2009;35:418-21.

34. Oostdam N, van Poppel MN, Wouters MG, et al. No effect of the FitFor2 exercise programme on blood glucose, insulin sensitivity, and birthweight in pregnant women who were overweight and at risk for gestational diabetes: results of a randomised controlled trial. BJOG 2012;119:1098-107.

35. Daly N, Farren M, McKeating A, et al. A Medically Supervised Pregnancy Exercise Intervention in Obese Women: A Randomized Controlled Trial. Obstet Gynecol 2017;130:1001-10.

36. Robinson HE, O'Connell CM, Joseph KS, et al. Maternal outcomes in pregnancies complicated by obesity. Obstet Gynecol 2005;106:1357-64.

37. Streuling I, Beyerlein A, Rosenfeld E, et al. Physical activity and gestational weight gain: a meta-analysis of intervention trials. BJOG 2011;118:278-84.

38. Du MC, Ouyang YQ, Nie XF, et al. Effects of physical exercise during pregnancy on maternal and infant outcomes in overweight and obese pregnant women: A meta-analysis. Birth 2019;46:211-21.

39. Sanabria-Martínez G, García-Hermoso A, Poyatos-León $\mathrm{R}$, et al. Effectiveness of physical activity interventions on preventing gestational diabetes mellitus and

Cite this article as: Xing Y, Wang X, Zhang W, Jiang H. The effect of exercise on maternal complications and birth outcomes in overweight or obese pregnant women: a meta-analysis. Ann Palliat Med 2020;9(6):4103-4112. doi: 10.21037/apm-20-2097 excessive maternal weight gain: a meta-analysis. BJOG 2015;122:1167-74.

40. Magro-Malosso ER, Saccone G, Di Mascio D, et al. Exercise during pregnancy and risk of preterm birth in overweight and obese women: a systematic review and meta-analysis of randomized controlled trials. Acta Obstet Gynecol Scand 2017;96:263-73.

41. Bain E, Crane M, Tieu J, et al. Diet and exercise interventions for preventing gestational diabetes mellitus. Cochrane Database Syst Rev 2015;(4):CD010443.

42. Oteng-Ntim E, Varma R, Croker H, et al. Lifestyle interventions for overweight and obese pregnant women to improve pregnancy outcome: systematic review and meta-analysis. BMC Med 2012;10:47.

43. Muktabhant B, Lawrie TA, Lumbiganon P, et al. Diet or exercise, or both, for preventing excessive weight gain in pregnancy. Cochrane Database Syst Rev 2015;(6):CD007145.

44. Domenjoz I, Kayser B, Boulvain M. Effect of physical activity during pregnancy on mode of delivery. Am J Obstet Gynecol 2014;211:401.e1-11.

45. Choi J, Fukuoka Y, Lee JH. The effects of physical activity and physical activity plus diet interventions on body weight in overweight or obese women who are pregnant or in postpartum: a systematic review and meta-analysis of randomized controlled trials. Prev Med 2013;56:351-64. 